\title{
How to Enhance Ipsilateral Actions of Pyramidal Tract Neurons
}

\author{
E. Jankowska, A. Cabaj, and L.-G. Pettersson \\ Department of Physiology, Göteborg University, 40530 Göteborg, Sweden
}

We have shown previously that ipsilateral pyramidal tract (PT) neurons facilitate the actions of reticulospinal neurons on feline motoneurons (Edgley et al., 2004), which indicates that they might assist the recovery of motor functions after injuries of contralateral corticospinal neurons. Nevertheless, stimulation of ipsilateral PT fibers alone only rarely evoked any synaptic actions in motoneurons. The aim of this study was to investigate possible ways of enhancing such actions and of inducing more effective excitation and inhibition of motoneurons. The effects of stimulation of the ipsilateral PT were investigated after eliminating the spinal actions of contralateral PT fibers by hemisecting the spinal cord at a low thoracic level and were estimated from intracellular records from hindlimb motoneurons. Two measures were used to enhance PT actions. The first was to increase the probability of activation of reticulospinal neurons by mutual facilitation of actions of ipsilateral and contralateral PT neurons. The second was to enhance synaptic transmission between PT neurons and reticulospinal neurons, and in pathways between the reticulospinal neurons and motoneurons via commissural interneurons, by systemic application of a $\mathrm{K}^{+}$channel blocker, 4-aminopyridine (4-AP). The results show that under favorable conditions, ipsilateral PT neurons may induce EPSPs and IPSPs in hindlimb motoneurons, or even action potentials, via the reticulospinal pathway. This study strengthens previous conclusions that ipsilateral PT neurons can potentially replace, at least to some extent, the actions of injured contralateral PT neurons. It also suggests that 4-AP might improve the progress of the recovery.

Key words: pyramidal tract; motor system; spinal cord; cat; 4-AP; interneurons

\section{Introduction}

Skilled voluntary movements depend to a great extent on corticospinal neurons; damage of these neurons (e.g., after stroke) may cause weakness or even paralysis (hemiparesis) on the opposite side of the body. Depending on the site and the degree of damage, some motor functions may recover, but the mechanisms through which this occurs are not fully understood. One mechanism could involve replacement of actions of the damaged neurons by actions of intact ipsilaterally projecting corticospinal neurons. This possibility has been considered repeatedly, although as reviewed by Hallett (2001), the conclusions of clinical studies regarding the role of ipsilateral corticospinal neurons in the recovery are inconsistent. In many studies, stimulation of the ipsilateral motor cortex failed to induce muscle contractions. This was the case with animals under barbiturate anesthesia [e.g., in studies by Woolsey's group (Welker et al., 1957)] or when the effects of transcranial magnetic stimulation were investigated on movements of hand muscles in healthy human subjects (Caramia et al., 1996; Turton et al., 1996; Netz et al., 1997); however, trans-

\footnotetext{
Received May 8, 2005; revised June 21, 2005; accepted June 22, 2005.

This work was supported by grants from National Institutes of Health (NS 40 863) and the Swedish Research Council (15393-01A). We thank Rauni Larsson for her invaluable assistance and Dr. S. A. Edgley and I. Hammar for their comments on this manuscript.

Correspondence should be addressed to E. Jankowska, Department of Physiology, Medicinaregatan 11, Box 432, 40530 Göteborg, Sweden. E-mail: elzbieta.jankowska@physiol.gu.se.

A. Cabaj's present address: Department of Neurophysiology, Nencki Institute of Experimental Biology, 02-093 Warszawa, Poland.

DOI:10.1523/JNEUROSCI.1838-05.2005

Copyright $\odot 2005$ Society for Neuroscience $\quad$ 0270-6474/05/257401-05\$15.00/0
}

cranial stimulation of the ipsilateral motor cortex induced contractions of proximal limb muscles, especially during bimanual phasic voluntary contractions (Bawa et al., 2004), and both proximal and hand muscles were activated in some hemiparetic patients (Caramia et al., 1996; Turton et al., 1996; Netz et al., 1997).

We recently presented evidence that ipsilateral pyramidal tract (PT) neurons potently facilitate disynaptic actions of reticulospinal (RS) neurons on motoneurons of both proximal and distal hindlimb muscles (Edgley et al., 2004) and might contribute to the movements of these muscles induced via extrapyramidal neuronal systems. One of the networks of the involved neurons is indicated in Figure 1, A and $B$. It includes reticulospinal neurons excited by PT fibers and spinal commissural interneurons, which may either excite or inhibit motoneurons. In this study, we investigated two possibilities for enhancing the actions mediated by this neuronal network. One was to take advantage of the convergence of both ipsilateral and contralateral PT neurons on reticulospinal neurons (Matsuyama and Drew, 1997; Kably and Drew, 1998) to increase the number of activated reticulospinal neurons by stimulating ipsilateral PT fibers at the background of subthreshold excitation of these neurons by contralateral PT stimulation, or vice versa, and thereby potentiate reticulospinal input to commissural interneurons. The second was to increase the effectiveness of synaptic transmission between PT and reticulospinal neurons and between the reticulospinal neurons and the commissural interneurons by a $\mathrm{K}^{+}$channel blocker, 4-aminopyridine (4-AP). 4-AP has been shown previously to be highly effective in spinal pathways (Jack et al., 1971; Jankowska et al., 1977) and is clinically beneficial (Nashmi and Fehlings, 2001). Both procedures were effective. 


\section{Materials and Methods}

Preparation. The experiments were performed on five deeply anesthetized cats weighing $2.6-4 \mathrm{~kg}$. All experimental procedures followed National Institutes of Health and European Union guidelines for animal care and were approved by a local ethical committee. Anesthesia was induced with sodium pentobarbital (40-44 mg/kg, i.p.) and maintained with intermittent doses of $\alpha$-chloralose ( $5 \mathrm{mg} / \mathrm{kg}$ administered every $1-2 \mathrm{~h}$, up to $55 \mathrm{mg} / \mathrm{kg}$, i.v.; Rhône-Poulenc Santé, Vitrysur-Seine, France). During recordings, neuromuscular transmission was blocked by pancuronium bromide $\left(\sim 0.2 \mathrm{mg} \cdot \mathrm{kg}^{-1} \cdot \mathrm{h}^{-1}\right.$, i.v.; Pavulon; Organon, Stockholm, Sweden), and the animals were artificially ventilated. Additional doses of $\alpha$-chloralose were given when increases in blood pressure or heart rate, which were monitored continuously, were evoked by peripheral or central stimulation or if the pupils dilated. Mean blood pressure was kept at $100-130 \mathrm{mmHg}$, and end-tidal concentration of $\mathrm{CO}_{2}$ was kept at $\sim 4 \%$ by adjusting the parameters of artificial ventilation and the rate of a continuous infusion of a bicarbonate buffer solution with 5\% glucose (1-2 $\left.\mathrm{mg} \cdot \mathrm{kg}^{-1} \cdot \mathrm{h}^{-1}\right)$. The core body temperature was kept at $\sim 37.5^{\circ} \mathrm{C}$ by servo-controlled infrared lamps. The experiments were terminated by a lethal dose of pentobarbital. In four experiments, 4-AP (Sigma, St. Louis, MO) was applied (0.2-0.4 $\mathrm{mg} / \mathrm{kg}$, i.v.).

The experimental procedures were as described by Edgley et al. (2004). Briefly, a laminectomy exposed the fourth to seventh lumbar (L4-L7), low thoracic (Th11-Th13), and third cervical (C3) spinal cord segments. The spinal cord was hemisected on the left side in the Th12 segment (after the dorsal columns were removed) to destroy connections between the crossed right pyramidal tract fibers and neurons on the left side of the lumbosacral enlargement. A number of peripheral nerves were dissected free, including the following: the quadriceps $(\mathrm{Q})$, sartorius (Sart), posterior bicepssemitendinosus (PBST), gastrocnemius-soleus (GS), and plantaris (PL).

The caudal part of the cerebellum was exposed by a craniotomy, and tungsten electrodes (impedance, $40-150 \mathrm{k} \Omega$ ) were placed in the right medial longitudinal fascicle (MLF) and in the left and right PTs. The electrodes were inserted at an angle of $35^{\circ}$ (with the tip directed rostrally). The initial targets were at the following Horsley-Clarke coordinates: P10 (where $\mathrm{P}$ equals posterior), R0.6 (where R equals right), $\mathrm{H}-5$ (where $\mathrm{H}$ equals horizontal) for MLF; P7, L1 (where L equals left), H - 10 and P5, $\mathrm{R} 1, \mathrm{H}-10$ for the left and right PTs, respectively. The final positions of the electrodes, however, were adjusted on the basis of records of descending volleys. The electrodes were kept at sites from which distinct descending volleys were evoked at stimulus intensities of $\leq 20 \mu \mathrm{A}$. At the end of the experiments, these sites were marked with electrolytic lesions. Their location was verified on $100-\mu \mathrm{m}$-thick frontal sections of the brainstem that were cut in the plane of insertion of the electrodes. As shown in Figure 1, $C$ and $D$, the MLF electrodes were placed at the level corresponding to the rostral border of the inferior olive; the PT electrodes were placed at the level of the caudal border of the trapezoid body.

Stimulation, recording, and analysis. Reticulospinal and PT fibers were stimulated with constant-current cathodal stimuli $(0.2 \mathrm{~ms} ; 50-200 \mu \mathrm{A})$. Descending volleys were recorded from the cord dorsum at a C3 segmental level and from the surface of the left lateral funiculus a few millimeters from the explored motor nuclei. As judged by these volleys, maximal MLF stimuli were 100-150 $\mu \mathrm{A}$, and maximal PT stimuli were 150-200 $\mu \mathrm{A}$. When maximal stimuli were used, we verified that no spread of current occurred between the PT and the MLF by comparing effects evoked from the optimal stimulation sites and from the sites $1-2 \mathrm{~mm}$ above or below them. Glass micropipettes filled with $2 \mathrm{~m}$ potassium citrate were used for intracellular records from motoneurons. Both the original data and averages of 10-20 single records were stored on-line. The areas and amplitudes of the potentials were estimated from the averaged records by using a software sampling and analysis system designed by E. Eide, T. Holmström, and N. Pihlgren (Göteborg University, Göteborg, Sweden). Differences between samples of neurons were assessed for statistical significance with Student's $t$ test.

\section{Results}

Mutual facilitation of actions evoked from the ipsilateral and contralateral corticospinal tracts via commissural interneurons and its enhancement by 4-AP

Figure $1 E-G$ shows that joint stimulation of the left and right PTs may evoke EPSPs in hindlimb motoneurons even when maximal stimuli applied separately to each PT are not effective. This procedure was successful in most motoneurons (55 of 66), whether in animals treated or not treated with 4-AP, although EPSPs evoked in this way before 4-AP administration usually did not exceed $0.2-0.3 \mathrm{mV}$ (see Fig. $4 G$ ).

Figure 2 illustrates the potent effects of the systemic application of 4-AP. Records in $A-D$ show that 4-AP enhanced the synaptic actions evoked from the left $(A)$ or the right $(B) \mathrm{PT}$, bringing out EPSPs (black traces) that PT stimuli alone failed to evoke before its application (gray traces). They also show that EPSPs appearing after 4 -AP were evoked at shorter latencies $(B, C)$ and of considerably larger amplitudes. These effects of 4-AP manifested themselves in parallel on actions evoked from the PT and from the MLF (Fig. 2D).

In the entire sample of 42 motoneurons recorded before the application of 4-AP (13 GS, 13 PBST, 3 PL, 7 Q, 5 Sart, and 1 unidentified), EPSPs from one PT were evoked in fewer than one-half and usually appeared only after a train of four to six 
A LPT $200 \mu \mathrm{A}$

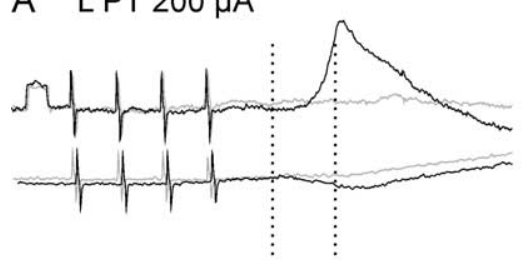

$\mathrm{B}$

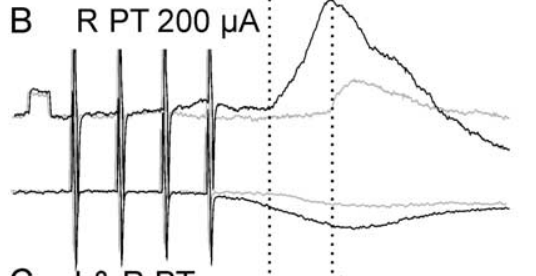

C L\&RPT
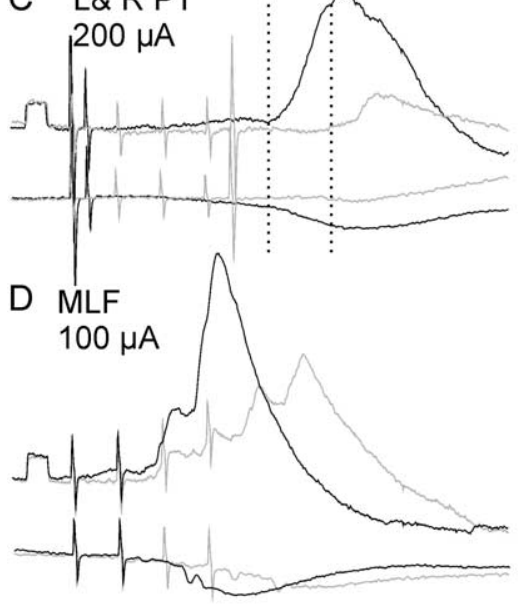

$0.2 \mathrm{mV}$

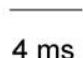

E LPT $150 \mu \mathrm{A}$

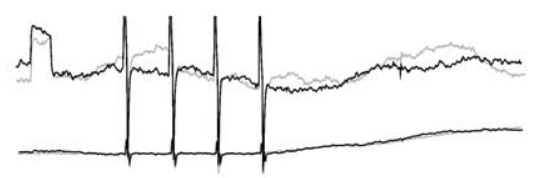

F $\quad$ R PT $150 \mu \mathrm{A}$

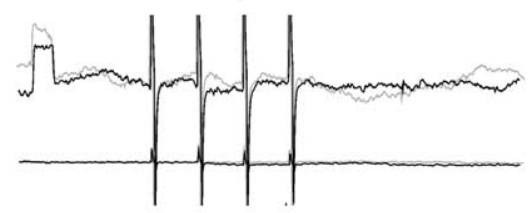

G

L \& R PT

$150 \mu \mathrm{A}$

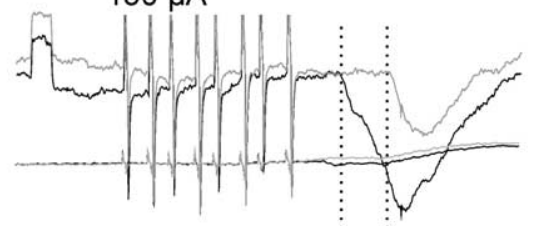

H L \& R PT $100 \mu \mathrm{A}$

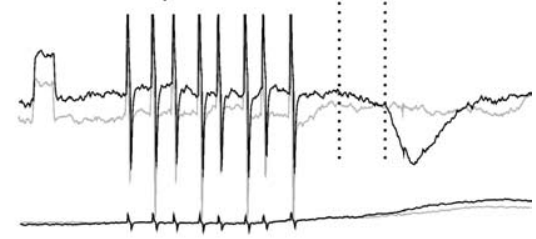

Before 4-AP

After 4-AP

Figure 2. Enhancement of excitatory synaptic actions from pyramids after 4-AP. Intracellular records from GS (A-D) and Sart $(\boldsymbol{E}-\boldsymbol{H})$ motoneurons (top traces) and from the cord dorsum (bottom traces). Gray traces are control records. Black traces are records taken after systemic application of 4-AP ( 0.33 and $0.2 \mathrm{mg} / \mathrm{kg})$; intracellular recordings were maintained from the same motoneurons. Dotted lines indicate onset of the earliest EPSPs or IPSPs evoked before and after 4-AP, respectively. Other indications are as in Figure 1.

stimuli, as in the sample of Edgley et al. (2004; their Fig. 1). After administration of 4-AP, EPSPs from one PT (either the left or the right) were induced in all of the 32 motoneurons tested ( 6 GS, 14 PBST, 2 PL, 2 Q, 7 Sart, and 1 unidentified) and by a smaller number of stimuli (one to three). Distinct EPSPs sometimes followed individual stimuli, as in the neuron illustrated in Figure $3 G-I$. Amplitudes of EPSPs evoked before 4-AP were $0.15 \pm 0.03$ and $0.12 \pm 0.02 \mathrm{mV}$ (mean $\pm \mathrm{SEM}$ ) from the left and right PTs, respectively. After administration of 4 -AP, they increased to $0.28 \pm 0.05$ and $0.26 \pm 0.05 \mathrm{mV}$. The changes were statistically significant $(p<0.02$ and $p<0.01)$.

The effectiveness of the mutual facilitation of actions of the left and right PTs after administration of 4-AP was also increased. In extreme cases, the combination of a single stimulus to both PTs sufficed (Fig. 2C, black trace) to evoke EPSPs, whereas before 4-AP administration, trains of stimuli applied to both PTs (Fig. 1C) or to at least one PT (Fig. 2C, gray trace) were needed. Larger EPSPs were evoked in this way in a much larger proportion of the motoneurons (Fig. 4, compare $H, J$ ), and their mean amplitude was increased from $0.44 \pm 0.09 \mathrm{mV}$ before 4 -AP to $0.99 \pm 0.1 \mathrm{mV}$ after 4-AP, with the difference statistically significant at $p<0.001$.

The enhancement of inhibitory PT actions was analyzed in mo- toneurons in which disynaptic IPSPs were evoked by stimulation of the MLF (two PBST, one GS, two Sart, and one unidentified motoneurons before 4-AP was given; one PBST, one PL, and seven Sart motoneurons after 4-AP was given). IPSPs appeared after joint stimulation of the left and right PTs in all of the motoneurons tested, even when stimuli applied to one $\mathrm{PT}$ alone were not effective, as illustrated in Figure $2 E-H$. Their amplitude was considerably increased after application of 4-AP (Fig. 2G) from the mean $0.14 \pm 0.04$ to $0.41 \pm 0.09 \mathrm{mV}, 0.17 \pm$ 0.03 to $0.46 \pm 0.14 \mathrm{mV}$, and $0.41 \pm 0.13$ to $0.78 \pm 0.09 \mathrm{mV}$ for IPSPs evoked from the left PT, right PT, and after joint stimulation of the left and right PTs, respectively. Their latencies shortened (Fig. 2G), and they were evoked at lower thresholds (Fig. $2 \mathrm{H}$ ) and by a smaller minimal number of stimuli (two to three instead of at least four stimuli were needed before 4-AP).

\section{Are PSPs evoked by ipsilateral PT neurons mediated exclusively by reticulospinal neurons?}

PSPs from the left and right PTs that are mediated via the pathways indicated in Figure 1, $A$ and $B$, would be expected to have similar latencies $\sim 1$ or 2 ms longer than latencies of PSPs evoked from the MLF; in Figure $4 A-C$, these fall between the gray and dotted lines. Minimal latencies of only approximately one-third of EPSPs evoked from the left and right PTs, or after their joint stimulation, were within the range of $4.4-5.5 \mathrm{~ms}$ from the effective stimuli and 1-1.5 ms longer than the latencies of EPSPs evoked from the MLF (3.8-4.2 ms); however, most EPSPs were evoked at latencies up to $2.5 \mathrm{~ms}$ longer (Fig. $4 A-C$, data points below the dotted lines). Minimal latencies of IPSPs (Fig. $4 A-C$, open symbols) were $0.3-0.6 \mathrm{~ms}$ longer than those of EPSPs but generally within the same range as for EPSPs; no statistically significant differences were found between latencies of EPSPs and IPSPs evoked from the same PT.

The difference between the minimal latencies of PSPs of PT and MLF origin of up to $1-1.5 \mathrm{~ms}$ corresponded to the difference between the positive peaks of the first (direct) component of the descending volleys from the right MLF (Fig. $3 A$ ) and of the second component of the $\mathrm{C} 4$ descending volleys from the left PT (Fig. 3B), which should reflect the activation of reticulospinal neurons by PT neurons (Edgley et al., 2004), both to be followed by activation of commissural interneurons. A similar delay was found in volleys recorded at a lumbar level (Fig. 3D-F) when PT stimuli applied after 4-AP were followed by distinct synchronized volleys (in two of four experiments in which the effects of 4-AP were tested).

EPSPs with latencies up to $\sim 1 \mathrm{~ms}$ longer were associated with the third component of the $\mathrm{C} 4$ descending PT volleys, which was delayed by $\sim 1 \mathrm{~ms}$ with respect to the second component. Figure $1 B$ illustrates the occurrence of the two MLF volleys, which followed the direct activation of MLF fibers and the subsequent activation of RS neurons via axon collaterals of other RS neurons. 
It also shows that the three PT volleys reflect direct activation of PT fibers and the subsequent activation of some RS neurons, first via axon collaterals of PT neurons and then via axon collaterals of other RS neurons.

Latencies of the remaining EPSPs, those shown above the dotted lines in Figure $4 A-C$, were longer. As a rule, longerlatency EPSPs were evoked when weaker stimuli or shorter trains of PT stimuli (Fig. $4 D, E)$ were used. The present material does not reveal how these long-latency EPSPs were mediated; however, their presence shows that other, longer routes between the ipsilateral corticospinal neurons and motoneurons are possible.

\section{Discussion}

The results of this study show that ipsilateral PT neurons can not only facilitate disynaptic actions of reticulospinal neurons but also use reticulospinal neurons as relay neurons to excite or inhibit spinal motoneurons and that ipsilateral and contralateral PT neurons can mutually enhance each others' actions by spatial facilitation. They also provide evidence that the actions of ipsilateral PT neurons may be greatly strengthened by pharmacologically increasing the effectiveness of synaptic transmission.

We interpret the synaptic actions of ipsilateral PT neurons on hindlimb motoneurons reported here as relayed by reticulospinal neurons, the reasons being as follows. First, because effects of stimulation of MLF and PT were qualitatively similar. Both EPSPs and IPSPs were evoked in parallel from MLF and after PT stimulation, and both were enhanced by 4-AP. Second, because the effects of stimulation of the left and right PTs and their mutual facilitation were similar when the actions of contralateral PT neurons were eliminated by hemisection of the spinal cord at a low thoracic level (Jankowska et al., 2003; Edgley et al., 2004). These similarities suggest that reticulospinal neurons are the main supraspinal relay neurons and commissural interneurons are the main spinal relay neurons of such actions; nevertheless, they do not exclude the involvement of other neurons, either supraspinal or spinal. For a more extensive discussion of the potential contribution of other relay neurons, see Edgley et al. (2004). The third reason for considering EPSPs and IPSPs found in this study as being relayed via reticulospinal neurons is the appropriate delay in PSPs after PT stimulation with respect to PSPs evoked from the MLF. The minimal differences between latencies of most of the EPSPs evoked from the MLF and from either the left or the right PT were within the same range as the delay of the first relayed PT volleys with respect to the direct MLF volleys.

PSPs that were evoked at latencies $\sim 1 \mathrm{~ms}$ longer might be attributed to the second rather than the first relayed PT volleys (Fig. 3B) and explained by disynaptically rather than monosynaptically evoked activation of reticulospinal neurons (Canedo and Lamas, 1993; Edgley et al., 2004). If the reticulospinal neurons were too weakly excited to be activated by nerve impulses in
R MLF $100 \mu \mathrm{A}$
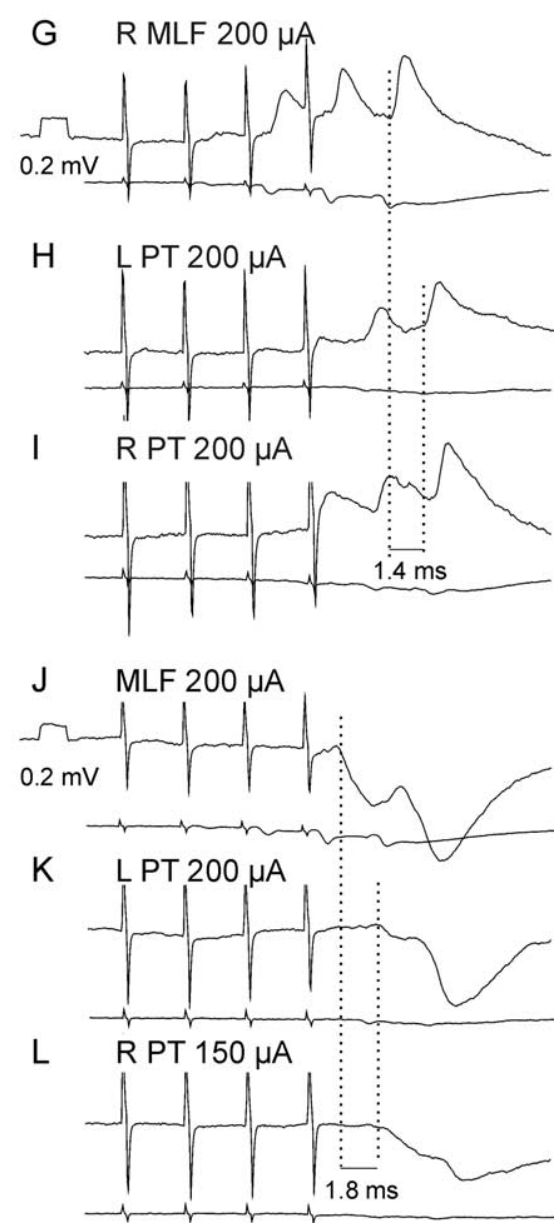

Figure 3. Relations between synaptic actions evoked from the MLF and from the left and right PTs. $\boldsymbol{A}-\boldsymbol{F}$, Descending volleys evoked by stimuli applied in the right MLF and in the left and right PTs. All were evoked by four stimuli, but only those after the fourth stimuli (at an expanded time base) are shown in $\boldsymbol{A}$ and $\boldsymbol{B}$. Arrows indicate direct volleys at the $\mathbf{C}$ segmental level that are first (direct) and the second and third (relayed) C3 PT volleys with respect to the direct MLF volleys. $\boldsymbol{D}-\boldsymbol{F}$, Dotted lines indicate (he first relayed PT and MLF volleys. $\mathbf{G}-\boldsymbol{L}$, Intracellular records from $\mathbf{Q}$ and Sart motoneurons (top traces) and records from the cord dorsum (bottom traces) in the same experiment. Note distinct EPSPs or IPSPs after individua second to fourth or third and fourth stimuli in $\mathbf{G}-\mathbf{I}$ and $\boldsymbol{J}-\mathbf{L}$, respectively. Note also the similar delays of both the relayed PT volleys and PSPs evoked by PT stimuli with respect to PSPs from the MLF.

collaterals of PT fibers, they might have required summation of the directly induced EPSPs with EPSPs evoked via other reticulospinal neurons (Fig. $1 B$ ). By following this line of reasoning, one might consider that the EPSPs evoked at even longer latencies were caused by polysynaptic activation of RS neurons via unknown supraspinal neuronal networks (reflected by later components of PT volleys); however, late PT actions could also be relayed via some polysynaptic pathways in the spinal cord. The latter could have been activated either by directly descending collaterals of the left and right PT neurons (Fig. $1 A$ ) or by spinal collaterals of reticulospinal neurons.

The two ways of enhancing the actions of ipsilateral PT neurons found to be effective in the present study might be useful for the recovery of motor functions after injuries of contralateral corticospinal neurons. The possibility of the mutual enhancement of actions of the left and right corticospinal neurons on reticulospinal neurons would depend on the level of the injury. When contralateral PT neurons are damaged at the level of the cortex or their axons are damaged rostral to where their brainstem collaterals are given off, the prospects of interactions between the left and right PT neurons 

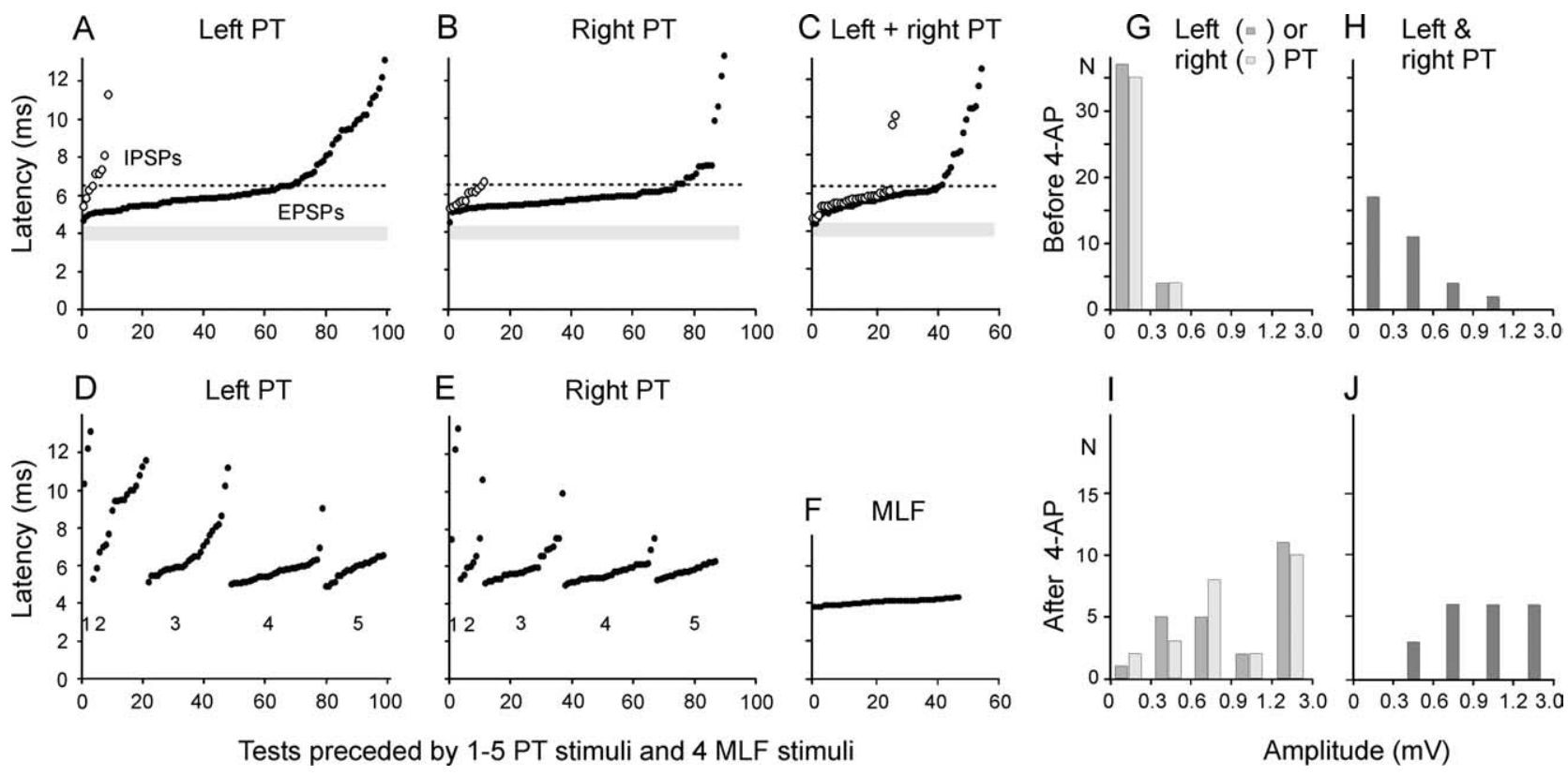

Figure 4. Latencies and amplitudes of EPSPs and IPSPs evoked from the left and right PTs. A-C, $F$, Latencies of EPSPs (filled symbols) and IPSPs (open symbols) evoked from the left and right PTs stimulated separately or jointly and from the MLF. The data points show the latencies of PSPs evoked in individual motoneurons by different parameters of stimuli (with 1-5 tests per motoneuron). They are ranked from the shortest to the longest. $\boldsymbol{D}, \boldsymbol{E}$, Data points in $\boldsymbol{A}$ and $\boldsymbol{B}$ are subdivided, being ranked for EPSPs evoked by different numbers of stimuli (indicated below). $\boldsymbol{A}-\boldsymbol{C}$, Thick gray lines indicate ranges of latencies of EPSPs evoked from the MLF. Dotted lines indicate latencies of PT EPSPS (up to $2.5 \mathrm{~ms}$ longer than EPSPs from the MLF) that are compatible with being mediated by either the first or the second relayed volleys illustrated in Figure 3B. No statistically significant differences were found between latencies of EPSPs or IPSPs evoked by joint ( $($ ) and either left or right PT stimulation. These were $6.42 \pm 0.25,6.72 \pm 0.9$, and $6.07 \pm 0.15 \mathrm{~ms}$ (mean $\pm \mathrm{SEM}$ ) from the effective stimulus, respectively. Differences between latencies of PSPs evoked from the left and right PTs were significant at $p<0.02$. G-J, Histograms of amplitudes of EPSPs evoked by trains of near-maximal PT stimuli (4-5 before and 3- 4 after application of 4-AP). Only motoneurons with action potentials $>45 \mathrm{mV}$ were used for the comparison. Student's $t$ test does not indicate statistically significant differences between effects from the left and right PTs in $\boldsymbol{G}$ and $I$ but does indicate highly statistically significant differences between effects evoked before and after application of 4-AP (see Results).

would be limited; however, the possibility of such an interaction should not be excluded when the injuries are not complete and the strengthening actions of the remaining neurons might make a difference in the process of recovery.

If contralateral PT neurons are damaged within the caudal part of the medulla or at a spinal level, the mutual enhancement of actions of the left and right PT neurons on reticulospinal neurons would be possible and might contribute to motor recovery, provided that more ventral reticulospinal pathways are not damaged at the same time. In both cases, enhancement of synaptic transmission at either supraspinal or spinal levels should be beneficial for the function of the remaining neuronal connections and for their plastic changes to occur. 4-AP has been used to treat patients with multiple sclerosis and after various spinal cord injuries and has been shown to improve motor performance (for review, see Nashmi and Fehlings, 2001). In patients with spinal cord injuries, the improvement after a single dose of 4-AP outlasted the time of its elimination (Hansebout et al., 1993), which might indicate plastic changes in the operation of the involved neuronal systems assisted by 4-AP. Knowing how ipsilateral PT neurons can contribute to the recovery of motor functions, and that 4-AP potentiates their actions, should encourage their maximal use during rehabilitation after injuries of contralateral PT neurons.

\section{References}

Bawa P, Hamm JD, Dhillon P, Gross PA (2004) Bilateral responses of upper limb muscles to transcranial magnetic stimulation in human subjects. Exp Brain Res 158:385-390.

Canedo A, Lamas JA (1993) Pyramidal and corticospinal synaptic effects over reticulospinal neurones in the cat. J Physiol (Lond) 463:475-489.

Caramia MD, Iani C, Bernardi G (1996) Cerebral plasticity after stroke as revealed by ipsilateral responses to magnetic stimulation. NeuroReport 7:1756-1760.
Edgley SA, Jankowska E, Hammar I (2004) Ipsilateral actions of feline corticospinal tract neurons on limb motoneurons. J Neurosci 24:7804-7813.

Hallett M (2001) Functional reorganization after lesions of the human brain: studies with transcranial magnetic stimulation. Rev Neurol (Paris) 157:822-826.

Hansebout RR, Blight AR, Fawcett S, Reddy K (1993) 4-Aminopyridine in chronic spinal cord injury: a controlled, double-blind, crossover study in eight patients. J Neurotrauma 10:1-18.

Jack JJ, Miller S, Porter R, Redman SJ (1971) The time course of minimal excitatory post-synaptic potentials evoked in spinal motoneurones by group Ia afferent fibres. J Physiol (Lond) 215:353-380.

Jankowska E, Lundberg A, Rudomin P, Sykova E (1977) Effects of 4 -aminopyridine on transmission in excitatory and inhibitory synapses in the spinal cord. Brain Res 136:387-392.

Jankowska E, Hammar I, Slawinska U, Maleszak K, Edgley SA (2003) Neuronal basis of crossed actions from the reticular formation on feline hindlimb motoneurons. J Neurosci 23:1867-1878.

Kably B, Drew T (1998) Corticoreticular pathways in the cat. I. Projection patterns and collateralization. J Neurophysiol 80:389-405.

Matsuyama K, Drew T (1997) Organization of the projections from the pericruciate cortex to the pontomedullary brainstem of the cat: a study using the anterograde tracer Phaseolus vulgaris-leucoagglutinin. J Comp Neurol 389:617-641.

Nashmi R, Fehlings MG (2001) Mechanisms of axonal dysfunction after spinal cord injury: with an emphasis on the role of voltage-gated potassium channels. Brain Res Brain Res Rev 38:165-191.

Netz J, Lammers T, Homberg V (1997) Reorganization of motor output in the non-affected hemisphere after stroke. Brain 120:1579-1586.

Turton A, Wroe S, Trepte N, Fraser C, Lemon RN (1996) Contralateral and ipsilateral EMG responses to transcranial magnetic stimulation during recovery of arm and hand function after stroke. Electroencephalogr Clin Neurophysiol 101:316-328.

Welker WI, Benjamin RM, Miles RC, Woolsey CN (1957) Motor effects of stimulation of cerebral cortex of squirrel monkey (Saimiri sciureus). J Neurophysiol 20:347-364. 